\title{
Erweitertes Zusammenwirken von natürlicher und künstlicher Intelligenz: Einführung in die Thematik
}

\author{
Reinhold Haux
}

\section{Zusammenfassung}

In praktisch allen Bereichen unserer Gesellschaften verändert die Digitalisierung unsere Lebenswelten. Dieses Buch befasst sich mit aktuellen Entwicklungen des Zusammenwirkens von natürlicher und künstlicher Intelligenz. Der Beitrag möchte in diese Thematik einführen und auf technische, ethische und rechtliche Herausforderungen des Zusammenwirkens lebender und nicht lebender Entitäten im Zeitalter der Digitalisierung hinweisen.

\section{Schlüsselwörter}

Zusammenwirken · Natürliche Intelligenz · Menschliche Intelligenz ·

Künstliche Intelligenz

Der Autor ist Sprecher der Kommission Synergie und Intelligenz der Braunschweigischen Wissenschaftlichen Gesellschaft. In diesen einführenden Bemerkungen berichtet der Autor über Arbeiten aus der Kommission Synergie und Intelligenz der Braunschweigischen Wissenschaftlichen Gesellschaft. An den Arbeiten der Kommission waren alle Kommissionsmitglieder beteiligt; besonders intensiv Klaus Gahl, Meike Jipp und Rudolf Kruse. Insofern ist dieser Beitrag zwar durch den Verfasser entstanden und dieser ist auch allein für dessen Inhalt verantwortlich. Es sei jedoch ausdrücklich erwähnt, dass an den Ergebnissen der Kommissionsarbeit alle Mitglieder - diese sind in Abschnitt 4 genannt beteiligt waren.

\section{R. Haux $(\bowtie)$}

Peter L. Reichertz Institut für Med. Informatik der TU Braunschweig und der Med. Hochschule Hannover, Braunschweig, Deutschland E-Mail: Reinhold.Haux@plri.de 


\section{$1 \quad$ Einleitung}

Es besteht mittlerweile ein vermutlich weltweiter Konsens darüber, dass die Digitalisierung erhebliche Veränderungen in praktisch allen Bereichen unserer Gesellschaften bewirkt und dass dadurch unsere gesamten Lebenswelten verändert werden. Dieses Buch befasst sich mit aktuellen Entwicklungen des Zusammenwirkens von natürlicher und künstlicher Intelligenz und daraus resultierenden Fragen, insbesondere für die Forschung. Das Buch steht in engem Zusammenhang $\mathrm{zu}$ den Arbeiten der Kommission Synergie und Intelligenz: technische, ethische und rechtliche Herausforderungen des Zusammenwirkens lebender und nicht lebender Entitäten im Zeitalter der Digitalisierung (kurz: Synergie und Intelligenz bzw. SYnENZ) der Braunschweigischen Wissenschaftichen Gesellschaft (BWG) (SYnENZ-Kommission 2019; BWG 2019).

Diese einführenden Bemerkungen verfolgen folgende Ziele, aus denen sich auch die Gliederung dieses Beitrags ergibt. Sie möchte

- über das Entstehen der SYnENZ-Kommission berichten (Abschn. 2),

- typische Fragen, die sich aus dem Zusammenwirken von natürlicher und künstlicher Intelligenz ergeben, nennen (Abschn. 3),

- die drei Dimensionen der Arbeit der SYnENZ-Kommission mit ihren Ausprägungen vorstellen (Abschn. 4) sowie

- auf die Notwendigkeit der inter- und multidisziplinären Bearbeitung des erweiterten Zusammenwirkens von natürlicher und künstlicher Intelligenz hinweisen (Abschn. 5).

\section{2 Über das Entstehen der SYnENZ-Kommission}

Der Autor dieser Einführung ist Medizininformatiker und auf diesem Gebiet in der Forschung tätig. Medizininformatik-Forschung befindet sich in der Spannung, zwei Ansprüchen gerecht zu werden: Sie soll sowohl originell (in der Methodik) als auch relevant (für den Menschen und dessen Gesundheitsversorgung) sein (van Bemmel 1996; Haux 2014, S. 260). Häufig sind die originellen und relevanten Medizininformatik-Forschungsthemen nur inter- und multidisziplinär zu bearbeiten (van Bemmel 2006).

Während der letzten Jahre haben sich die Themen, die der Autor in seiner Forschung bearbeitet, erheblich verändert. Konzentrierten sich diese lange Zeit und durchaus gut begründet auf Informationssysteme des Gesundheitswesens und deren Funktionalitäten, Architekturen und Infrastrukturen (vgl. Haux 2006, 2013) 
so befassen sich die Arbeiten nun in verstärktem Maße mit assistierenden Gesundheitstechnologien, mit Informatik-Diagnostika und Informatik-Therapeutika bis hin zu intelligenten Wohnungen, die als Diener der Bewohner zur Gesundheitsversorgung in Prävention, Diagnostik, Therapie und Nachsorge beitragen sollen (vgl. Haux 2016, 2017; Haux et al. 2016a, b). Diese Entwicklungen gehen einher mit zunehmender Digitalisierung der Lebenswelten in praktisch allen Gesellschaften dieser Welt und mit zunehmend verfügbarer Künstlicher Intelligenz, letzteres aufgrund der heute vorhandenen (UN 2015, Punkt 15) Möglichkeiten der Erfassung, Repräsentation und Analyse von Daten durch funktional umfassende, leistungsfähige und vernetzte Maschinen.

Am 7. Februar 2018 befasste sich die BWG auf Initiative des Neuroinformatikers und Robotikers Jochen Steil in ihrem 14. Bioethik-Symposium mit der Thematik Roboter im Operationssaal (Gahl 2019; Steil et al. 2019). Chirurgen und Robotiker, Ethiker, Informatiker und Juristen, befassten sich mit den Auswirkungen des zunehmenden Einsatzes von Robotik und Digitaltechnologie im Operationssaal. Diskutiert wurde über die systemischen Veränderungen aus Sicht von Patienten, Ärzten und Operationsteams sowie über die Frage, wer letztendlich bei einer Operation entscheidet. Ist es wirklich (noch) der Chirurg alleine? Inwieweit entscheiden OP-Roboter als funktional umfassende Maschinen mit und, falls ja, welche Konsequenzen ergeben sich daraus?

Schon während der im Jahr 2017 begonnenen Vorbereitungen des 14. Bioethik-Symposiums regte der damalige BWG-Präsident Otto Richter an, eine Kommission zur Bearbeitung dieser sowohl für die Gesellschaft relevante als auch für die Forschung originelle Thematik einzurichten und fragte den Verfasser, ob er hier aktiv werden könnte. So wichtig das Thema Roboter im Operationssaal war, so klar wurde in den vorbereitenden Gesprächen, dass diese Thematik als eine Instanz eines umfassenderen Themenkomplexes gesehen und bearbeitet werden sollte: dem erweiterten Zusammenwirken von natürlicher und künstlicher Intelligenz.

Es bildete sich die Kommission Synergie und Intelligenz: technische, ethische und rechtliche Herausforderungen des Zusammenwirkens lebender und nicht lebender Entitäten im Zeitalter der Digitalisierung (SYnENZ-Kommission 2019), die im Jahr 2017 ihre Arbeit aufnahm (SYnENZ-Jahresbericht 2017, 2018, 2019). Abb. 1 enthält das Logo der SYnENZ-Kommission in deutscher und englischer Sprache.

Dieses Buch basiert in weiten Teilen auf den Ausarbeitungen von Vorträgen des 1. BWG Symposiums über das erweiterte Zusammenwirken von natürlicher und künstlicher Intelligenz (SYnENZ-Symposium 2019). Das SYnENZ-Symposium fand am 14. und 15. März 2019 statt. Es wurde von Mitgliedern der SYnENZKommission initiiert und durch diese organisiert. 


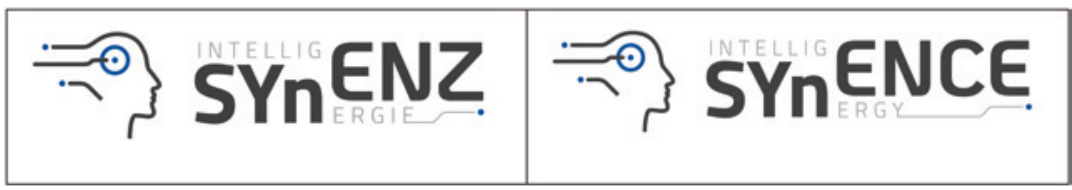

Abb. 1 Logo der SYnENZ-Kommission deutsch und englisch. (Quelle: SYnENZKommission 2019)

\title{
3 Typische Fragen zum Zusammenwirken von natürlicher und künstlicher Intelligenz
}

Die auf der Presseinformation zu dem SYnENZ-Symposium genannten, an die Öffentlichkeit gerichteten Fragen waren:

\begin{abstract}
Was ist Künstliche Intelligenz und was bedeutet sie für uns Menschen? Wie verändert sie Gesundheitsversorgung, Mobilität und Landwirtschaft? Wird in der Arztpraxis eine intelligente Maschine Symptome erfragen und Diagnosen erstellen? Wie verändern autonome Fahrzeuge den Straßenverkehr für Fußgänger und Radfahrer? Falls ja: Geht das überhaupt - technisch, ethisch, juristisch? Und die wichtigste Frage: Wollen wir dies eigentlich? (TU Braunschweig 2019).
\end{abstract}

Die in der Einführung zu dem SYnENZ-Symposium einleitend genannten Fragen lauteten:

- Wie wird Zusammenleben in Zeiten zunehmender Digitalisierung in Zukunft aussehen?

- Welche Synergien ergeben sich durch das nun mögliche erweiterte Zusammenwirken von Menschen, Tieren und Pflanzen einerseits und von Maschinen andererseits, in anderen Worten durch ein solch erweitertes Zusammenwirken von natürlicher und künstlicher Intelligenz?

- Können wir zwischen bloß zeitgemäßen und angemessenen Formen des Zusammenwirkens unterscheiden?

- Und können, um angemessene Formen zu erreichen und um zwar zeitgemäße, aber problematische Formen zu vermeiden, Empfehlungen gegeben werden?

- Lässt sich der Grad des Zusammenwirkens bestimmen? 


\section{$4 \quad$ Die drei Dimensionen der Arbeit der SYnENZ- Kommission}

Die SYnENZ-Kommission befasst sich in grundsätzlicher Weise mit den sich durch die zu Beginn genannten Entwicklungen ergebenden neuen Formen des Zusammenlebens. Dabei soll es nicht nur um neue Kommunikations- und Interaktionsformen zwischen Menschen gehen. Auch das erweiterte Zusammenwirken mit nicht lebenden Objekten (Maschinen) oder mit anderen Lebewesen, in welcher Art auch immer, ist Gegenstand der Kommissionsarbeit.

Die nachfolgend in Abb. 2 genannten Themen bilden die inhaltlichen Schwerpunkte für gemeinsame interdisziplinäre Forschung. Kriterien für deren Auswahl waren Originalität, Relevanz und das Potenzial, diese durch Mitglieder der Kommission gut bearbeiten zu können. Die Inhalte sind in drei Anwendungsbereiche gegliedert. Diese sollten keinesfalls als getrennte und getrennt bearbeitbare Themenbereiche, sondern vielmehr als Koordinaten - semantische Bezugssysteme - eines dreidimensionalen Raumes gesehen werden.

Anwendungsgebiete erweiterten Zusammenwirkens:

- erweitertes Zusammenwirken in Medizin und Gesundheitsversorgung

- erweitertes Zusammenwirken mit Tieren und Pflanzen in der Landwirtschaft

- physische und virtuelle Mobilitätsformen im erweiterten Zusammenwirken

ethisch-rechtliche Aspekte im erweiterten Zusammenwirken ...

- zu Autonomie und Verantwortung

- zu Individualität und Kollektivität

- zu Individualisierung und Normierung

methodisch-technische Aspekte des erweiterten Zusammenwirkens ...

- zu maschineller und menschlicher Intelligenz und zur Robustheit

- zur Kooperation mittels Schwarm-Intelligenz

- bei der Evaluation dieses Zusammenwirkens

Abb. 2 Die drei Dimensionen der Arbeit der SYnENZ-Kommission mit ihren Ausprägungen. (Quelle: SYnENZ-Jahresbericht 2019) 
Die Beiträge in diesem Buch orientieren sich an dieser Strukturierung. Fragen in Bezug auf die ethisch-rechtlichen Aspekte sind:

- bei Individualität und Kollektivität: Wird es, beispielsweise durch intensivierte Kommunikation, durch die erweiterte Nutzung von Assistenzsystemen oder durch zusätzliche Implantate zu einem verstärkten kollektiven Zusammenwirken von Individuen kommen? Wie kann diese Kollektivität aussehen? Welche Bedeutung hat sie für die Individualität?

- bei Individualisierung und Normierung: Ist das $\mathrm{zu}$ erwartende erweiterte Zusammenwirken förderlich für die individuelle Entwicklung von Entitäten, insbesondere von Menschen? Oder birgt erweitertes Zusammenwirken, beispielsweise durch die Nutzung von maschinellen Systemen zu Entscheidungsunterstützung, das Risiko der Normierung menschlichen Verhaltens und persönlicher Entwicklung in sich? Wie kann Individualisierung gefördert und Normierung verhindert werden?

- bei Autonomie und Verantwortung: Welche Konsequenzen ergeben sich aus dem erweiterten Zusammenwirken insbesondere von Menschen einerseits und Maschinen andererseits für die Autonomie von (menschlichen) Entscheidungen und für die damit verbundene Übernahme von Verantwortung? Dies besonders bei Maschinen mit hoher Funktionalität, umfassender Sensorik bzw. Aktorik und/oder der Fähigkeit der Analyse großer Datenbestände?

Um trotz der hohen Komplexität dieser Thematik zu möglichst konkreten Aussagen zu kommen, werden diese Aspekte vor allem in Bezug auf die genannten drei Anwendungsgebiete behandelt. Neben eher klassischen methodisch-technischen Themen wurde bewusst die Evaluation erweiterten Zusammenwirkens auch als methodisches Thema mit aufgenommen und bearbeitet.

\section{$5 \quad$ Notwendigkeit inter- und multidisziplinärer Bearbeitung}

Angemessene Formen der heute schon vorhandenen und zukünftig zu erwartenden technischen Möglichkeiten des erweiterten Zusammenwirkens von natürlicher und künstlicher Intelligenz können nur gemeinsam mit der Behandlung damit verbundener ethischer und rechtlicher Fragen sinnvoll erarbeitet werden. Diese Überzeugung der Notwendigkeit einer inter- und 
multidisziplinären Bearbeitung teilt der Autor mit den anderen Mitgliedern der SYnENZ-Kommission. Das SYnENZ-Symposium hat die Notwendigkeit dieser Sichtweise klar bestätigt. Und auch dieses Buch spiegelt diese Auffassung mit seinen Autorinnen und Autoren aus Informatik, Ingenieurwissenschaften, Jura, Psychologie, Medizin, Philosophie und weiteren Fachgebieten und mit den teilweise inter- und multidisziplinär verfassten Beiträgen wider.

Abschließend seien noch die Namen der Mitglieder der SYnENZ-Kommission genannt (Stand: November 2019). Susanne Beck (Rechtswissenschaften), Klaus Gahl (Medizin), Reinhold Haux (Medizinische Informatik, Sprecher), Engel Hessel (Digitale Landwirtschaft), Meike Jipp (Human Factors, stellvertretende Sprecherin), Nicole Karafyllis (Philosophie), Joachim Klein (Chemie), Ralf Kreikebohm (Sozial- und Arbeitsrecht), Rudolf Kruse (Computational Intelligence, stellvertretender Sprecher), Karsten Lemmer (Verkehr und Energie), Otto Luchterhandt (Öffentliches Recht), Michael Marschollek (Medizinische Informatik), Sanaz Mostaghim (Intelligente Systeme), Otto Richter (Agrarökologie), Kerstin Schwabe (Experimentelle Neurochirurgie), Jochen Steil (Robotik), Lars Wolf (Connected and Mobile Systems), Klaus-Hendrik Wolf (Medizinische Informatik).

\section{Literatur}

BWG. (2019). Braunschweigische Wissenschaftliche Gesellschaft (BWG). https://bwg-nds. de. Zugegriffen: 1. Nov. 2019.

Gahl, K. (2019). Roboter im Operationssaal. Bericht über das 14. Bioethik-Symposium der BWG in Zusammenarbeit mit der TU Braunschweig. Erscheint in Braunschweigische Wissenschaftliche Gesellschaft (Hrsg.), Jahrbuch 2018. Braunschweig: J. Cramer. https://bwg-nds.de/veröffentlichungen-jahrbuch-und-abhandlungen/. Zugegriffen: 1. Nov. 2019.

Haux, R. (2006). Health information systems - Past, present, future. International Journal of Medical Informatics, 75, 268-281.

Haux, R. (2013). „Wer waren Deine wichtigsten Lehrer?. GMS Medizinische Informatik, Biometrie und Epidemiologie, 9(3), Doc15. https://doi.org/10.3205/mibe000143. URN: urn:nbn:de:0183-mibe0001434.

Haux, R. (2014). On determining factors for good research in biomedical and health informatics. IMIA Yearbook of Medical Informatics, 9, 255-264, Diskussion S. 265-72.

Haux, R. (2016). My Home is my hospital. On recent research on health-enabling technologies. Studies in Health Technology and Informatics, 226, 3-8. 
Haux, R. (2017). On informatics diagnostics and informatics therapeutics - Good medical informatics research is needed here. Studies in Health Technology and Informatics, 238, 3-7.

Haux, R., Koch, S., Lovell, N. H., Marschollek, M., Nakashima, N., \& Wolf, K.-H. (2016). Health-enabling and ambient assistive technologies: Past, present, future. IMIA Yearbook of Medical Informatics, 25(Suppl. 1), 76-91.

Haux, R., Marschollek, M., \& Wolf, K.-H. (2016). Über assistierende Gesundheitstechnologien und neue Formen kooperativer Gesundheitsversorgung durch Menschen und Maschinen. In A. Manzeschke, \& F. Karsch (Hrsg.), Roboter, Computer und Hybride. Was ereignet sich zwischen Menschen und Maschinen? (S. 131-143). Baden-Baden: Nomos.

Steil, J., Finas, D., Beck, S., Manzeschke, A., \& Haux, R. (2019). Robotic systems in operating theatres: New forms of team-machine interaction in health care. On challenges for health information systems on adequately considering hybrid action of humans and machines. Methods of Information in Medicine, 58, e14-e25.

SYnENZ-Jahresbericht. (2017). Jahresbericht 2017 der BWG-Kommission erweitertes Zusammenwirken lebender und nicht lebender Entitäten - Technische, ethische und rechtliche Herausforderungen im Zeitalter der Digitalisierung. In Braunschweigische Wissenschaftliche Gesellschaft (Hrsg.) (2018), Jahrbuch 2017 (S. 208-209). Braunschweig: J. Cramer. https://bwg-nds.de/veröffentlichungen-jahrbuch-und-abhandlungen/. Zugegriffen: 1. Nov. 2019.

SYnENZ-Jahresbericht. (2018). Jahresbericht 2018 der BWG-Kommission Synergie und Intelligenz: Technische, ethische und rechtliche Herausforderungen des Zusammenwirkens lebender und nicht lebender Entitäten im Zeitalter der Digitalisierung (SYnENZ). Braunschweigische Wissenschaftliche Gesellschaft (Hrsg.) (2019), Jahrbuch 2018 (S. 231-234). Braunschweig: J. Cramer. https://bwg-nds.de/veröffentlichungen-jahrbuch-und-abhandlungen/. Zugegriffen: 1. Nov. 2019.

SYnENZ-Jahresbericht. (2019). Jahresbericht 2019 der BWG-Kommission Synergie und Intelligenz: Technische, ethische und rechtliche Herausforderungen des Zusammenwirkens lebender und nicht lebender Entitäten im Zeitalter der Digitalisierung (SYnENZ). Erscheint in Braunschweigische Wissenschaftliche Gesellschaft (Hrsg.) (2020), Jahrbuch 2019. Braunschweig: J. Cramer. http://bwg-nds.de/veröffentlichungen-jahrbuch-und-abhandlungen/. Zugegriffen: 1. Nov. 2019.

SYnENZ-Kommission. (2019). Kommission Synergie und Intelligenz (SYnENZ) der Braunschweigischen Wissenschaftlichen Gesellschaft. https://bwg-nds.de/ kommissionen/kommission-synenz/. Zugegriffen: 1. Nov. 2019.

SYnENZ-Symposium. (2019). Zusammenwirken von natürlicher und künstlicher Intelligenz. 1. BWG-Symposium über das erweiterte Zusammenwirken lebender und nicht lebender Entitäten im Zeitalter der Digitalisierung, 14.-15.2.2019, Braunschweig. https://bwg-nds.de/kommissionen/kommission-synenz/. Zugegriffen: 1. Nov. 2019.

TU Braunschweig. (2019). Presseinformation der Technischen Universität Braunschweig vom 13.2.2019. Wie künstliche Intelligenz unser Leben verändert. https://magazin. tu-braunschweig.de/pi-post/wie-kuenstliche-intelligenz-unser-leben-veraendert/. Zugegriffen: 1. Nov. 2019. 
UN 2015. Transforming our world: The 2030 Agenda for Sustainable Development. Resolution 70/1, von der Vollversammlung der Vereinten Nationen angenommen am 25 September 2015. https://www.un.org/en/development/desa/population/migration/ generalassembly/docs/globalcompact/A_RES_70_1_E.pdf. Zugegriffen: 1. Nov. 2019.

van Bemmel, J. H. (1996). Medical informatics, art or science? Methods of Information in Medicine, 35, 157-172, Diskussion S. 173-201.

van Bemmel, J. H. (2006). The young person's guide to biomedical informatics. Methods of Information in Medicine, 45, 671-680.

Open Access Dieses Kapitel wird unter der Creative Commons Namensnennung 4.0 International Lizenz (http://creativecommons.org/licenses/by/4.0/deed.de) veröffentlicht, welche die Nutzung, Vervielfältigung, Bearbeitung, Verbreitung und Wiedergabe in jeglichem Medium und Format erlaubt, sofern Sie den/die ursprünglichen Autor(en) und die Quelle ordnungsgemäß nennen, einen Link zur Creative Commons Lizenz beifügen und angeben, ob Änderungen vorgenommen wurden.

Die in diesem Kapitel enthaltenen Bilder und sonstiges Drittmaterial unterliegen ebenfalls der genannten Creative Commons Lizenz, sofern sich aus der Abbildungslegende nichts anderes ergibt. Sofern das betreffende Material nicht unter der genannten Creative Commons Lizenz steht und die betreffende Handlung nicht nach gesetzlichen Vorschriften erlaubt ist, ist für die oben aufgeführten Weiterverwendungen des Materials die Einwilligung des jeweiligen Rechteinhabers einzuholen.

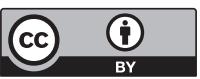

